\title{
Identification of Neuregulin-2 as a novel stress granule component
}

\author{
Jin Ah Kim ${ }^{1, \#}$, Aravinth Kumar Jayabalan ${ }^{1, \#}$, Vinoth Kumar Kothandan ${ }^{1}$, Ramesh Mariappan ${ }^{1}$, Younghoon Kee ${ }^{2}$ \\ $\mathcal{E}$ Takbum Ohn ${ }^{1, *}$ \\ ${ }^{1}$ Department of Cellular \& Molecular Medicine, College of Medicine, Chosun University, Gwangju 61452, Korea, ${ }^{2}$ Department of Cell \\ Biology, Microbiology, and Molecular Biology, College of Arts and Sciences, University of South Florida, Tampa, Florida 33620, USA
}

Stress Granules (SGs) are microscopically visible, phase dense aggregates of translationally stalled messenger ribonucleoprotein (mRNP) complexes formed in response to distinct stress conditions. It is generally considered that SG formation is induced to protect cells from conditions of stress. The precise constituents of SGs and the mechanism through which SGs are dynamically regulated in response to stress are not completely understood. Hence, it is important to identify proteins which regulate SG assembly and disassembly. In the present study, we report Neuregulin-2 (NRG2) as a novel component of SGs; furthermore, depletion of NRG2 potently inhibits SG formation. We also demonstrate that NRG2 specifically localizes to SGs under various stress conditions. Knockdown of NRG2 has no effect on stress-induced polysome disassembly, suggesting that the component does not influence early step of SG formation. It was also observed that reduced expression of NRG2 led to marginal increase in cell survival under arsenite-induced stress. [BMB Reports 2016; 49(8): 449-454]

\section{INTRODUCTION}

Stress granules (SGs) are membrane-less, dense cytoplasmic aggregates formed in response to various environmental stress conditions (1). In general, cells monitor adverse conditions with the help of at least four stress sensing kinases such as (a) PKR (Protein Kinase R) activated by viral infection, heat shock and UV irradiation, (b) PERK (PKR like Endoplasmic Reticulum Kinase) activated by unfolded protein response, (c) GCN2 (General Control Non-derepressible 2) induced by starvation

*Corresponding author. Tel: +82-62-230-6285; Fax: +82-62-2336337; E-mail: tohn@chosun.ac.kr

${ }^{\#}$ These authors contributed equally to this work.

http://dx.doi.org/10.5483/BMBRep.2016.49.8.090

Received 3 June 2016, Revised 14 June 2016, Accepted 21 June 2016

Keywords: NRG2, RNA granules, Stress granules, Stress signaling, Translation and (d) HRI (Heme Regulated Initiation factor 2 kinase) triggered by sodium arsenite exposure. Activation of either of these kinases causes phosphorylation of elF $2 \alpha$ at serine 51 , a component of elF2/tRNAi/GTP ternary complex which is required for translation initiation. In absence of ternary complex, translation initiation is compromised, thus leading to formation of translationally stalled mRNP complex, which eventually accumulates into SGs (2). Thus, SGs mostly harbor translation initiation factors such as elF3, elF4A, elF4G, elF4E as well as poly(A)-mRNA, PABP and small (40S) but not large (60S) ribosomal proteins. In addition, many RNA binding proteins (RBPs) such as TIA-1, TIAR, G3BP and FMRP and some signaling proteins such as RACK1, TRAF2 are present in SGs (3). Processing bodies (PBs) are another type of RNA granule which closely resembles SGs but are functionally and compositionally different. SGs appear only after a cell senses stress stimuli, whereas PBs are present in normal (unstressed) cells and proportionally increase upon stress exposure. PB mostly harbors mRNA decay enzymes such as Dcp1 or Hedls, DCP2, etc. Under certain stress conditions, SG and PB come close together and dock some proteins and mRNA. By this phenomenon, these two compartments decide the cell's fate under stress conditions by allowing translation of few transcripts while some are stored (in SGs) or degraded (in PBs).

Stress-induced translational repression and SG assembly allows cell to reprogram overall gene expression profile and permit selective translation of stress responsive genes. SGs are classified as canonical (or universal) and non-canonical, based on their composition, structure or size and number of granules per cell; however, until date no functional difference has been reported between them. SGs usually assemble within minutes after encountering condition of stress, but the disassembling kinetics differs with type of stress. There are numerous factors that are involved in the regulation of SG assembly such as RNA-binding proteins, post-translational modifications, presence of PRD (prion-related domain), low complexity (LC) or intrinsically disordered (ID) regions in SG nucleating factors (1, 4). Although mechanism of SG assembly is essential for cell survival, it has also been implicated in many disease conditions such as cancer, fragile $\mathrm{X}$ syndrome and neurodegenerative diseases such as ALS and AD (5). Most of the SG

ISSN: 1976-670X (electronic edition)

Copyright (c) 2016 by the The Korean Society for Biochemistry and Molecular Biology

(c) This is an open-access article distributed under the terms of the Creative Commons Attribution Non-Commercial License (http://creativecommons.org/licenses/by-nc/4.0) which permits unrestricted non-commercial use, distribution, and reproduction in any medium, provided the original work is properly cited. 
associated diseases are reported as a consequence of its abnormal aggregation, which fail to disassemble even after cell's recovery from stress. Deregulation of proteins involved in assembly or disassembly kinetics (precisely proteins involved in the stress signaling pathway) has been proposed as a probable mechanism for failure of granules to disassemble $(6,7)$. There have been several studies explaining the role of SG formation, components and function, but information about proteins modulating SG dynamics is deficient.

We have previously identified several genes, which are involved in the regulation of SG and processing body based on an RNAi screen (8). In quest of the signaling protein involved in SG assembly, we reviewed our data and found NRG2 was one of the hits from the screen. Neuregulins (NRGs) are a member of epidermal growth like (EGF-like) factor, which stimulates ErbB-receptor tyrosine phosphorylation and mediates distinct biological processes. NRG2, a splice variant of NRG1 is a transmembrane protein, which helps in regulation of cell proliferation, differentiation, survival; whereas deregulation of NRG2 results in diseases such as cancer, schizophrenia and Alzheimer's disease $(9,10)$. Activation of NRG2 signal by ErbB receptors can elicit different downstream signaling pathways such as MAPK, PI3, Protein Kinase C and JAK-STAT pathway (11). Nevertheless, data about how these signals provoke specific cellular responses such as proliferation or survival is limited, except for a report, which identified an intracellular signaling component, MEMO (Mediator of ErbB2-driven cell motility) and reported its integration with ErbB signal and control over cell motility (12). It has also been reported that NRG2 crosstalk with broad range of intracellular signaling networks in which ErbB receptor acts as a scaffold in transmitting the information to downstream cellular pathways (13).

In this report, we identify neuregulin-2 (NRG2) as a novel component of SGs. Depletion of NRG2 strongly inhibits SG aggregation, but does not affect stress-induced polysome disassembly. We also found that NRG2 localized to SGs under multiple stress condition. Moreover, reduced expression of NRG2 led to marginal increase in cell survival under arsenite-induced stress suggesting that the component may negatively regulate stress-mediated cell death.

\section{RESULTS}

SiRNA mediated NRG2 knockdown impairs SG assembly Previous RNAi-mediated loss-of-function screen has identified NRG2 as a potential regulator of SGs $(8,14)$. To test whether NRG2 indeed is a component and / or regulator of SG assembly, we first depleted endogenous NRG2 using two different siRNA's and evaluated SG assembly kinetics. SiCONT (non-targeting) and siNRG2 knocked-down cells were cultured in the absence or presence of sodium arsenite to induce SG assembly in a time dependent manner (30 and $60 \mathrm{~min}$ ). Immunofluorescence (IF) microscopic analysis was performed subsequently employing universal SG marker (elF3b) and SG/PB marker (RCK) to visualize the presence of SG and PB, respectively. As shown in Fig. 1A, depletion of NRG2 strongly impaired SG formation after exposure to arsenite as compared to siCONT cells. Immunoblot analysis confirmed significant reduction in the expression of NRG2 (Fig. 1B). Conversely, the percentage of RCK-positive cells remained unchanged in the NRG2 knockdown cells, suggesting no effect of NRG2 depletion on $\mathrm{PB}$ assembly. Our results reveal that the percentage of PB in basal (around 63\% unstressed cells) and after stress $(98 \%)$ followed similar assembly pattern as comparable to earlier reports $(15,16)$. We tested this phenomenon in different cell lines including U2OS stably expressing EGFP-G3BP (Fig. 1C), HEK293T, HeLa and

A

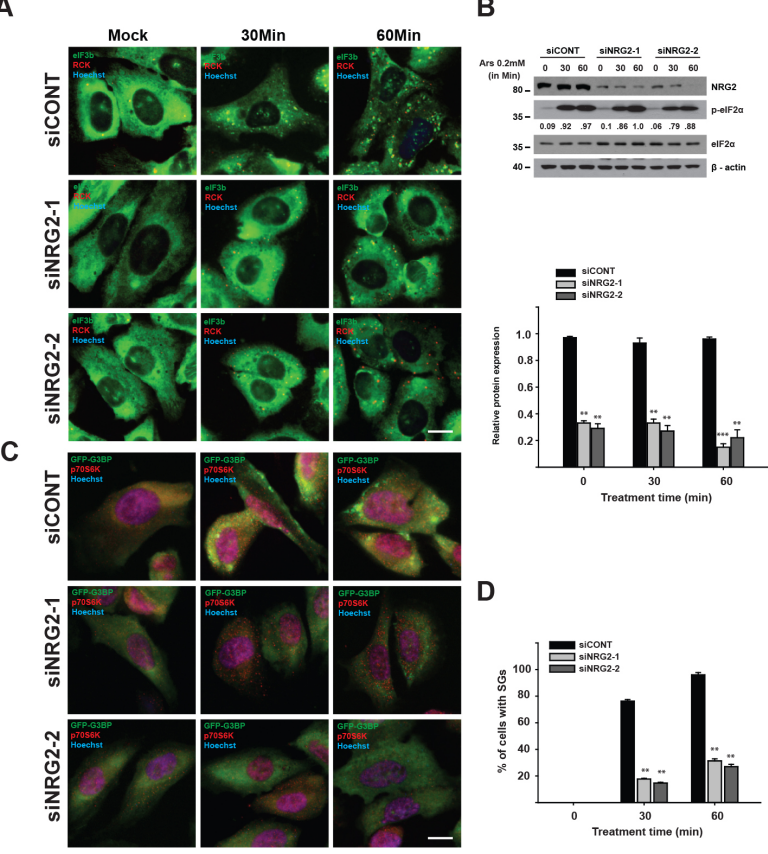

Fig. 1. NRG2 knockdown impairs SGs assembly. (A) U2OS cells transfected with siCONT, siNRG2-1 and siNRG2-2 for $90 \mathrm{hrs}$ were grown on coverslips and treated with $0.2 \mathrm{mM}$ arsenite for indicated time points. Cells were then stained against universal SG marker elF3b (green), SG/PB marker RCK (red) and nucle stain Hoechst (blue). (B) Whole cell lysates were subjected to immunoblot assay to determine knockdown efficiency of NRG2-1 and NRG2-2 and induction of p-elF2alpha. Actin was used as loading control. Quantified western blot results are represented below. (C) U2OS derived EGFP-G3BP stable cell line was transfected with different siRNAs and subjected to arsenite treatment as shown in Fig. 1A. Presence of SG and PB was then visualized using GFP fluorescence and antibody reactive to p70S6K, respectively. (D) SG quantification data showing percentage of cells with SGs. Data are means \pm s.d. of at least three independent experiments. ${ }^{*} P<0.05$; ${ }^{* * P}<0.01$; ***P< 0.001 , Student's t-test. Scale bar, $10 \mu \mathrm{m}$. 
obtained consistent results (data not shown). Moreover, siNRG2 treated cells exhibited $70 \%$ inhibition of SGs even after $60 \mathrm{~min}$ of exposure to arsenite with respect to siCONT cells (Fig. 1D), suggesting that NRG2 plays a direct potential role at early stage of SG assembly (8).

\section{NRG2 localizes to SGs under arsenite-induced stress}

The proteins involved in the regulation of SG assembly such as translation initiation factors, RNA binding proteins, kinases or stress signaling molecules generally localizes to SG after encountering stress stimuli. NRG2 is a transmembrane protein with $\mathrm{N}$-terminal sequence present in the extracellular region and the C-terminal portion protruding through the cytoplasm. Based on the location of NRG2, we also investigated whether the transmembrane protein re-localizes to SGs under arseniteinduced stress (10). In brief, immunofluorescence microscopy was employed for U2OS cells treated with $0.5 \mathrm{mM}$ sodium arsenite and stained with antibodies against elF3b (SG marker), NRG-2 and p70S6K (PB marker). As shown in Fig. 2A, NRG2 strongly localized to SGs (elF3b) after arsenite treatment,
A

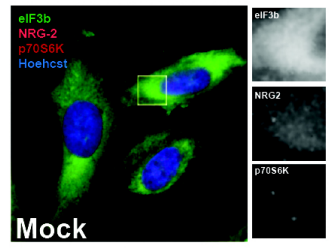

B

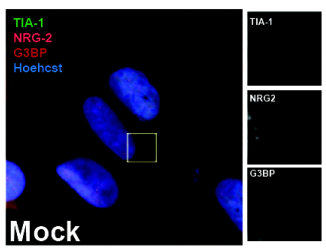

C

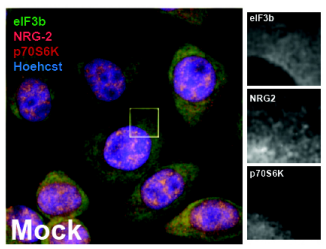

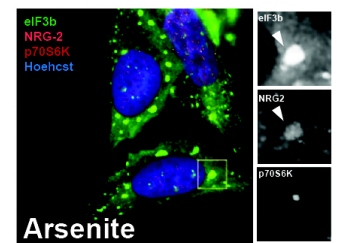
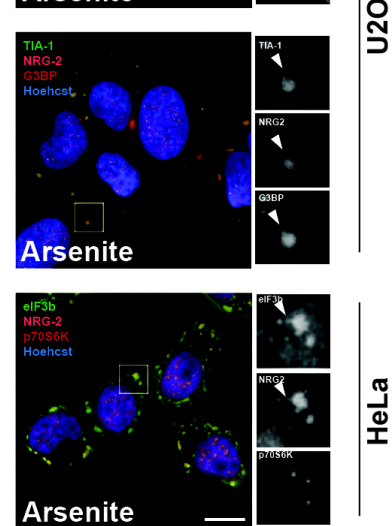

Fig. 2. NRG2 localizes to SGs under arsenite-induced stress. (A) U2OS cells grown on coverslips were treated with $0.5 \mathrm{mM}$ arsenite for $1 \mathrm{hr}$ before processing for immunostaining against elF3b (green), NRG2 (red), PB marker S6K (far red) and nuclei stain Hoechst (blue). (B) U2OS cells were treated with $0.5 \mathrm{mM}$ arsenite for $1 \mathrm{hr}$ and stained against established SG markers like TIA (green), NRG2 (red), G3BP (far red) and nuclei stain Hoechst (blue). (C) HeLa cells were grown on coverslips and treated with $0.5 \mathrm{mM}$ arsenite for $1 \mathrm{hr}$ prior to immunostaining against elF3b (green), NRG2 (red), S6K (far red) and nuclei stain Hoechst (blue). Scale bar, $10 \mu \mathrm{m}$. Enlarged views of boxed areas show individual channels (green, red and far-red). Arrow head represents SGs. Data presented represents at least three independent experiments. whereas no localization to PBs (p70S6K) was observed. This result is consistent with the observation that NRG2 knockdown specifically affects SG formation, but not the PB assembly (Fig. 1) (17). Localization of NRG2 to SGs was further verified by counter-staining with other well characterized SG markers such as TIA-1 and G3BP (Fig. 2B) (18). Localization of NRG2 to SGs was also observed in HeLa (Fig. 2C) and HEK293T cells (data not shown). SGs generally recruit specific proteins at different stages of aggregation, for instance, elF3b and G3BP readily accumulate in the SG at primary stage as soon as cells encounter condition of stress; whereas other proteins such as RACK1, TRAF2 localize to SG at the stage of secondary aggregation $(2,19,20)$. Our assays show that NRG2 localizes to the SGs as early as $15 \mathrm{~min}$ after stress induction (data not shown) in a manner similar to TIA-1/TIAR suggesting that NRG2 may play a role in the initial aggregation of SGs (19). Consistently, NRG2 knockdown exhibited its impact on the initial formation of SGs. This observation implies two possible roles of NRG2, either it might act as a stress signaling protein for granule assembly or may play a role in the primary aggregation stage. Collectively, these data suggest that NRG2 is a bonafide component of SG as well as a regulator of $\mathrm{SG}$ assembly.

\section{Depletion of NRG2 does not affect the stress induced polysome disassembly}

It is well known that the stress-induced phosphorylation of elF2 $\alpha$ acts as an initial stimulus for SG assembly (19). The phosphorylated elF2 $\alpha$ eventually causes translational arrest and polysome disassembly. To examine whether NRG2 has a role in polysome disassembly under arsenite-induced stress, we depleted endogenous NRG2 and performed the polysome profiling analysis. U2OS cells transfected with siCONT or siNRG2, were treated with or without $0.2 \mathrm{mM}$ arsenite for 30 min. Cells were subsequently lysed and the extracted proteins were subjected to ribosomal fractionation. Unexpectedly, the control and NRG2 knockdown cells did not show any differences in the disassembly profile upon arsenite treatment (Fig. 3). This result is a reminiscence of previous finding that O-GlcNAc depletion did not affect polysome disassembly but exhibited its effect only on SG aggregation (21). Altogether, this data suggests that NRG2 acts downstream of p-elF2 $\alpha$ and may act as a nucleation factor for SG assembly.

\section{NRG2 depletion enhances cell survival under condition of stress}

The major functional role for SG formation is to protect cells from adverse stress stimuli by temporarily halting energetically costly translation process and activate survival program. This allows cells to conserve much cellular resources, and thereby recover from stress with easiness $(22,23)$. The polysome profile data suggested that NRG2 acts independently or at a level downstream of the elF $2 \alpha$ phosphorylation. Recent report identified that inhibition of SG assembly induces stress- 

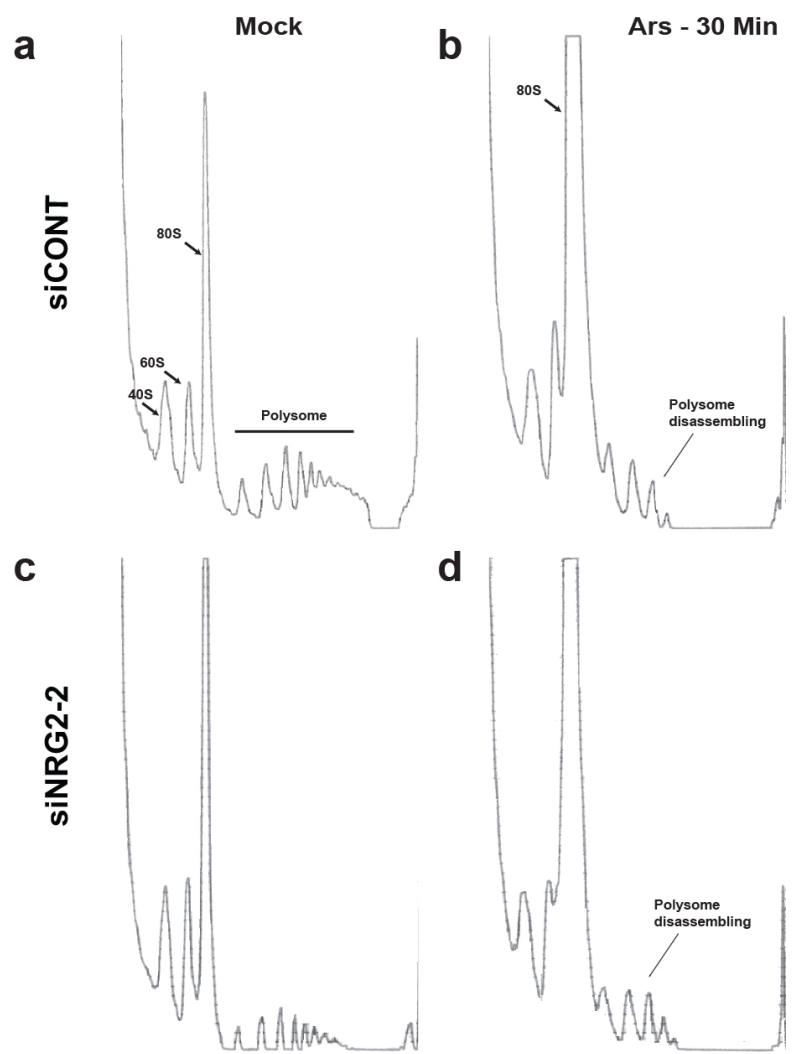

Fig. 3. NRG2 depletion does not affect stress induced polysome disassembly. U2OS cells seeded at low density in $150 \mathrm{~mm}$ dish were knocked-down twice with siCONT or siNRG2-1 for $90 \mathrm{hrs}$. Cells were then untreated (control) or treated with $0.2 \mathrm{mM}$ arsenite for $30 \mathrm{~min}$ to induce polysome disassembly. After treatment, cells were lysed in polysome lysis buffer, subjected to sucrose gradient and polysome profile analysis. See Methods for detailed procedure.

mediated apoptosis regardless of p-eIF $2 \alpha$ activation. As we have shown earlier (Fig. 1), NRG2 knockdown strongly impaired SG assembly, so we sought to determine to what extent this phenotype affects cell viability rate under arsenite-induced stress. Henceforth, we carried out the cell survival assay with siCONT, siNRG2-1 and siNRG2-2 transfected cells following $0.5 \mathrm{mM}$ arsenite treatment at different time points. To our surprise, the NRG2-depleted cells showed modest resistance to the arsenite treatment as compared to control cells (Fig. 4). Particularly, this phenomenon was more evident after 2 hrs of arsenite $(0.5 \mathrm{mM})$ exposure, and the conditions were respective dose and time at which stress-induced apoptotic pathway triggers $(24,25)$. This result suggests that NRG2 promotes cell death upon arsenite-induced stress. Indeed, some reports suggest that NRGs are involved in apoptosis $(26,27)$. Based on these results, we propose a model stating that NRG2 is recruited to

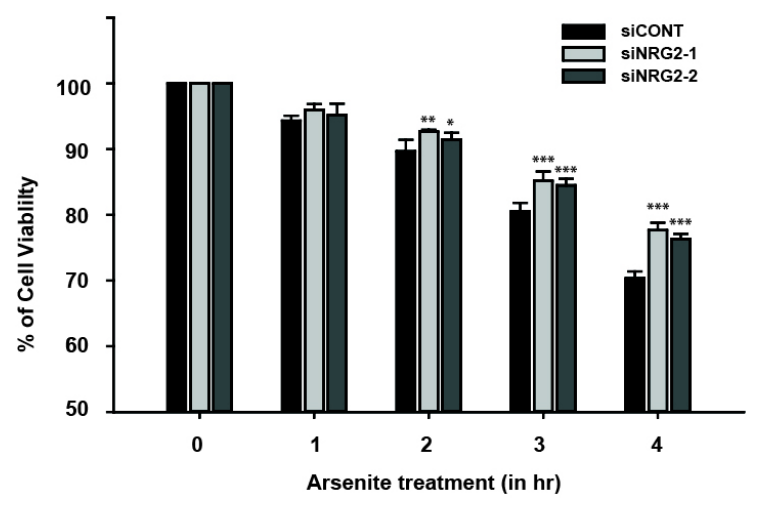

Fig. 4. NRG2 knockdown increases cell survival rate upon oxidative stress. U2OS cells transfected with control siRNA or two different siRNAs targeting NRG2 for 90 hrs were seeded in 96 well plate. Next day, cells were left untreated or treated with $0.5 \mathrm{mM}$ arsenite at different time point $(0,1,2,3$ and $4 \mathrm{hrs})$. Cells were then subjected to cell survival assay as described in Methods. The results are mean \pm S.D of atleast three independent experiments. ${ }^{*} \mathrm{P}<0.05 ;{ }^{*} * \mathrm{P}<0.01 ; * * * \mathrm{P}<0.001$, Student's t-test.

SGs at initial stage to prevent stress-induced apoptotic pathway under condition of mild stress (until $2 \mathrm{hrs}$ ); whereas, under prolonged stress (after 2 hrs) NRG2 eventually re-localizes from SGs to participate in stress mediated apoptosis.

\section{DISCUSSION}

Stress induced translation arrest and reprogramming of gene expression is a surveillance mechanism adopted by cells to survive under conditions of stress as well as save energy to cope up during the process of stress recovery. Recruitment of untranslated housekeeping but not stress-responsive mRNAs into SGs with the help of numerous RNA binding proteins, signaling molecules and subsequent docking with $\mathrm{PBs}$ is critical to decide cell fate and maintain RNA triage. In the present work, we report NRG2 (neuregulin-2) as a novel component of SGs. We show that NRG2 depletion leads to impairment in SG assembly dynamics, without affecting PB assembly. Our further analysis reveals that NRG2 does not affect polysome disassembly suggesting its independent action or immediate downstream of elF2 $\alpha$ phosphorylation likely at the aggregation stage similar to O-GlcNAc modification (8).

NRG2 is also known to mediate ErbB receptor tyrosine kinase signaling (10). Whereas elF2 $\alpha$ phosphorylation is critical for translation arrest and ribosome run-off, SG components such as RNA binding proteins (TIA, HuR, etc.) predominantly act as nucleation (or aggregation) factor. Recently, it was revealed that many signaling proteins recruited to SGs typically harbor prion related (PR), intrinsically disordered (ID) or low complexity domain (LCD). These structurally disordered domains which fail to fold into 
active tertiary structure have been demonstrated to be involved in phase separation and formation of membrane-less compartments inside cell. Thus, we checked whether any disordered region resides in NRG2 using the web server (http://iupred.enzim.hu/) and surprisingly most of the Cterminal region was found to be completely disordered (28). It confirms our hypothesis that NRG2 acts as an aggregation factor to facilitate SG assembly after p-elF2 $\alpha$ (Fig. 3). The cytoplasmic C-terminal domain of NRG2 is also involved in trafficking and proteolytic processing by undergoing dimerization, thus suggesting its involvement in phase separation after stress signaling.

It is stated that depletion of key components of SGs concomitantly reduces the survival rate after stress exposure (29). Typically, this phenomenon is due to inhibition of SG assembly; however, we observed a marginal increase in survival rate with NRG2 knockdown. It is also known that SGs inhibit stress induced apoptosis by recruiting signaling molecules such as RACK1, TRAF2 under conditions of mild stress, but it is also apparent that these proteins localize to SG at later stage. In fact, it is clear that LCD regions present in NRG2 can act in the nucleation process at the primary aggregation stage and eventually localize to SGs within minutes after stress exposure. One likely reason for late localization of RACK1 to SGs is that, the signaling molecule is completely devoid of any disordered region. This suggests that the disordered region present in NRG2 is an advantageous criterion for SG assembly, unless further experiments addressing the role of NRG2 deletion mutants and its localization to SG come into scenario. The finding is analogous to the report stating SG induced suppression of stress-responsible, MAPK pathway by actively recruiting RACK1 under mild conditions of stress. Moreover, the obtained data adds NRG2 to the previously proposed model of SGs as a multi-cellular signaling hub, where many signaling proteins orchestrate stress induced response (1).

\section{MATERIALS AND METHODS}

\section{Cell culture and transfection}

The U2OS (human osteosarcoma), HeLa and HEK293T cells were maintained in DMEM medium (Welgene) supplemented with $10 \%$ inactivated FBS (Welgene), $1 \%(\mathrm{v} / \mathrm{v})$ penicillin and streptomycin (Lonza) at $37^{\circ} \mathrm{C}$ in $5 \% \mathrm{CO}_{2}$. Transfection of siRNA was performed using Lipofectamine 2000 (Invitrogen) at $40 \mathrm{nM}$ final concentration as per manufacturer's instruction. siRNA sequences were as follows: siCONT, $5{ }^{`}$-GCATTCA CTTGGATAGTAA-3`; siNRG2-1, $5{ }^{`}$-GGAACAGCCCTTAGTC TTT-3`; siNRG2-2, $5{ }^{`}$-GGTCGGGTGGCGTTGGTAA-3` .

\section{Western blot analysis}

Cells were lysed in RIPA buffer (50 mM Tris-Cl (pH 8.0), 150 $\mathrm{mM} \mathrm{NaCl}, 0.1 \%$ SDS, $1 \%$ NP-40, $1 \mathrm{mM}$ EDTA, $1 \%$ Sodium deoxycholate, containing proteinase inhibitors $5 \mathrm{mM} \mathrm{NaF}, 1 \mathrm{mM}$
PMSF) for $15 \mathrm{~min}$ in ice and centrifuged at 13,000 rpm for 15 min. Proteins were quantified using Bradford reagent. Total proteins $(20-50 \mu \mathrm{g})$ were subjected to SDS-PAGE, transferred to nitrocellulose membranes and detected with respective antibodies. Western blot was performed using ECL detection system. Primary antibodies were purchased as follows: Anti $\beta$-actin from Abcam (Cambridge, MA, USA); p-elF2 $\alpha$ from Biomol (Farmingdale, NY, USA); elF2 $\alpha$ from Santa Cruz Biotechnology and anti - NRG2 from Millipore.

\section{Immunofluorescence analysis}

Cells grown on coverslips were either untreated or treated with arsenite, rinsed twice with PBS (pH7.4), fixed with paraformaldehyde for $15 \mathrm{~min}$, permeabilized with cold methanol for 10 min and blocked in 5\% normal horse serum in PBS containing $0.02 \%$ sodium azide for $1 \mathrm{~h}$. Primary antibodies diluted in blocking solution were added to the cells and incubated either at RT for $1 \mathrm{~h}$ or overnight at $4^{\circ} \mathrm{C}$. Cells were then washed with PBS (three times, 10 min each) and incubated with respective secondary antibodies for $1 \mathrm{~h}$ at RT, washed thrice with PBS (10 min each) and were mounted in polyvinyl medium. Fluorescence microscopic images were taken using a Nikon Eclipse 80i fluorescence microscope (40X). All images were processed in Image $\mathrm{J}$ and compiled in Adobe Photoshop CS5. Primary antibodies for ICC were purchased as follows: elF3b, G3BP, TIA-1 and p70S6 kinase from Santa Cruz; RCK from Bethyl laboratories and NRG2 from Millipore. Cy2-, Cy3-, Cy5- conjugated secondary antibodies were purchased from Jackson Immunoresearch labs.

\section{Polysome profiling}

U2OS cells $(2 \times 150 \mathrm{~mm}$ dish) were treated with indicated concentration of sodium arsenite for indicated time. After treatment, $10 \mu \mathrm{g} \mathrm{ml}^{-1}$ cycloheximide was added and incubated for $5 \mathrm{~min}$ at RT, washed with cold PBS, then lysed in $1 \mathrm{ml}$ of polysome lysis buffer (20 mM HEPES (pH7.6), $5 \mathrm{mM}$ $\mathrm{MgCl}_{2}, 125 \mathrm{mM} \mathrm{KCl}, 1 \% \mathrm{NP}-40,2 \mathrm{mM}$ DTT) supplemented with $100 \mu \mathrm{g} \mathrm{ml}^{-1}$ cycloheximide (Sigma), protease inhibitor cocktail (EDTA-free; pierce) and RNAsin (Ambion) in a cold room. Cell lysates were tumbled for $15 \mathrm{~min}$ at $4^{\circ} \mathrm{C}$ and centrifuged at 13,000 rpm for $15 \mathrm{~min}$. The supernatants were fractionated in $17.5-50 \%$ linear sucrose gradients by ultracentrifugation (35,000 rpm for 2 h $40 \mathrm{~min}$ ) in a Beckman ultracentrifuge using SW40-Ti rotor. Gradients were eluted with a gradient fractionator (Brandel) and monitored with a UA-5 detector (ISCO). Fractions were acetone precipitated at $-20^{\circ} \mathrm{C}$ for overnight and processed for further analysis.

\section{Cell viability assay}

Cell viability was determined using MTT [3-(4,5-Dimethylthiazol-2-yl)-2,5-diphenyltetrazolium bromide] (Sigma). After treatment, cells were washed with PBS twice and incubated with $0.5 \mathrm{~g} / \mathrm{ml} \mathrm{MTT}$ for $2 \mathrm{hrs}$ at $37^{\circ} \mathrm{C}$ until the formation of dark formazan crystals. After lysis with DMSO, absorbance 
was measured at $570 \mathrm{~nm}$. All measurements were done in triplicate wells.

\section{ACKNOWLEDGEMENTS}

This study was supported by research fund from Chosun University, 2015 (K206207004).

\section{REFERENCES}

1. Kedersha N, Ivanov P and Anderson P (2013) Stress granules and cell signaling: more than just a passing phase? Trends Biochem Sci 38, 494-506

2. Anderson P and Kedersha N (2008) Stress granules: the Tao of RNA triage. Trends Biochem Sci 33, 141-150

3. Stoecklin G and Kedersha N (2013) Relationship of GW/P-bodies with stress granules. Adv Exp Med Biol 768, 197-211

4. Ohn T and Anderson P (2010) The role of posttranslational modifications in the assembly of stress granules. Wiley Interdiscip Rev RNA 1, 486-493

5. Ramaswami M, Taylor JP and Parker R (2013) Altered ribostasis: RNA-protein granules in degenerative disorders. Cell 154, 727-736

6. Buchan JR, Kolaitis RM, Taylor JP and Parker R (2013) Eukaryotic stress granules are cleared by autophagy and Cdc48/VCP function. Cell 153, 1461-1474

7. Molliex A, Temirov J, Lee J et al (2015) Phase separation by low complexity domains promotes stress granule assembly and drives pathological fibrillization. Cell 163, 123-133

8. Ohn $\mathrm{T}$, Kedersha $\mathrm{N}$, Hickman $\mathrm{T}$, Tisdale $\mathrm{S}$ and Anderson P (2008) A functional RNAi screen links O-GlcNAc modification of ribosomal proteins to stress granule and processing body assembly. Nat Cell Biol 10, 1224-1231

9. Chang H, Riese DJ 2nd, Gilbert W, Stern DF and McMahan UJ (1997) Ligands for ErbB-family receptors encoded by a neuregulin-like gene. Nature 387, 509-512

10. Carraway KL 3rd, Weber JL, Unger MJ et al (1997) Neuregulin-2, a new ligand of ErbB3/ErbB4-receptor tyrosine kinases. Nature $387,512-516$

11. Falls DL (2003) Neuregulins: functions, forms, and signaling strategies. Exp Cell Res 284, 14-30

12. Marone R, Hess D, Dankort D, Muller WJ, Hynes NE and Badache A (2004) Memo mediates ErbB2-driven cell motility. Nat Cell Biol 6, 515-522

13. Britsch S (2007) The neuregulin-I/ErbB signaling system in development and disease. Adv Anat Embryol Cell Biol 190, 1-65

14. Buchan JR and Parker R (2009) Eukaryotic stress granules: the ins and outs of translation. Mol Cell 36, 932-941
15. Parker R and Sheth U (2007) P bodies and the control of mRNA translation and degradation. Mol Cell 25, 635-646

16. Decker CJ and Parker R (2012) P-bodies and stress granules: possible roles in the control of translation and mRNA degradation. Cold Spring Harb Perspect Biol 4, a012286

17. Shah KH, Zhang B, Ramachandran V and Herman PK (2013) Processing body and stress granule assembly occur by independent and differentially regulated pathways in Saccharomyces cerevisiae. Genetics 193, 109-123

18. Tourriere H, Chebli K, Zekri L et al (2003) The RasGAPassociated endoribonuclease G3BP assembles stress granules. J Cell Biol 160, 823-831

19. Kedersha NL, Gupta M, Li W, Miller I and Anderson P (1999) RNA-binding proteins TIA-1 and TIAR link the phosphorylation of elF-2 alpha to the assembly of mammalian stress granules. J Cell Biol 147, 1431-1442

20. Arimoto K, Fukuda H, Imajoh-Ohmi S, Saito H and Takekawa M (2008) Formation of stress granules inhibits apoptosis by suppressing stress-responsive MAPK pathways. Nat Cell Biol 10, 1324-1332

21. Li CH, Ohn T, Ivanov P, Tisdale $S$ and Anderson $P$ (2010) elF5A promotes translation elongation, polysome disassembly and stress granule assembly. PLoS One 5, e9942

22. Hofmann S, Cherkasova V, Bankhead P, Bukau B and Stoecklin G (2012) Translation suppression promotes stress granule formation and cell survival in response to cold shock. Mol Biol Cell 23, 3786-3800

23. Anderson $P$ and Kedersha N (2009) Stress granules. Curr Biol 19, R397-398

24. Huang S, Huang CF and Lee T (2000) Induction of mitosis-mediated apoptosis by sodium arsenite in HeLa S3 cells. Biochem Pharmacol 60, 771-780

25. Cai B and Xia Z (2008) p38 MAP kinase mediates arsenite-induced apoptosis through FOXO3a activation and induction of Bim transcription. Apoptosis 13, 803-810

26. Daly JM, Jannot CB, Beerli RR, Graus-Porta D, Maurer FG and Hynes NE (1997) Neu differentiation factor induces ErbB2 down-regulation and apoptosis of ErbB2-overexpressing breast tumor cells. Cancer Res 57, 3804-3811

27. Kirchhoff $\mathrm{S}$ and Hauser H (1999) Cooperative activity between HER oncogenes and the tumor suppressor IRF-1 results in apoptosis. Oncogene 18, 3725-3736

28. Dosztanyi Z, Csizmok V, Tompa P and Simon I (2005) IUPred: web server for the prediction of intrinsically unstructured regions of proteins based on estimated energy content. Bioinformatics 21, 3433-3434

29. Eisinger-Mathason TS, Andrade J, Groehler AL et al (2008) Codependent functions of RSK2 and the apoptosispromoting factor TIA-1 in stress granule assembly and cell survival. Mol Cell 31, 722-736 